\title{
Troponin Levels and Outcomes in Patients with Embolic Stroke of Undetermined Source
}

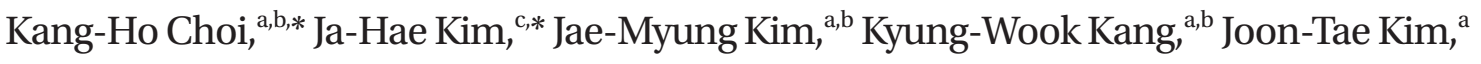 \\ Seong-Min Choi, ${ }^{a}$ Man-Seok Park, ${ }^{a} \mathrm{Ki}-H y u n \mathrm{Cho}^{\mathrm{a}}$ \\ ${ }^{a}$ Department of Neurology, Chonnam National University Hospital, Chonnam National University Medical School, Gwangju, Korea \\ ${ }^{b}$ Department of Neurology, Chonnam National University Hwasun Hospital, Hwasun, Korea \\ cMolecular Imaging Center, Department of Nuclear Medicine, Chonnam National University Hospital, Chonnam National University Medical \\ School, Gwangju, Korea
}

*These authors contributed equally to the manuscript as first author.

\section{Dear Sir:}

A new clinical construct of embolic stroke of undetermined source (ESUS) has been proposed to group heterogeneous patients with embolism of undetermined cause despite recommended diagnostic workup. ${ }^{1}$ Although the emboli in ESUS may originate from various potential embolic sources (PESs), cardioembolic sources may account for most PESs in ESUS.' Cardiac troponin (cTn) is a sensitive and specific marker of cardiac dysfunction. ${ }^{2}$ Recent studies have suggested that elevated cTn levels are more common in patients with ESUS than in those with noncardioembolic stroke. ${ }^{3}$ Elevated cTn levels may be associated with worse clinical outcomes and a higher risk of vascular events after stroke. ${ }^{4,5}$ Therefore, we investigated the effectiveness of conventional cTn I (cTnl) and high-sensitivity cTn $\mathrm{T}$ (hs-cTnT) levels in predicting clinical outcomes in patients with ESUS.

This single-center retrospective cohort study used a prospective registry. Subjects were divided into normal and high troponin groups according to sex-specific 99th percentile upper reference limits (Supplementary Figure 1). The primary outcome measure was the first occurrence of major adverse cerebrovascular and cardiovascular events (MACCE) according to the baseline cTn levels over a 1-year period after ESUS. The secondary outcomes included constituents of MACCE. We enrolled 1,838 consecutive patients with ESUS admitted to our center (Supplementary Figure 1). The baseline patient characteristics and annual number of patients in the cTnl and hs-cTnT groups are presented in Supplementary Tables 1-4. Elevated cTnl and hs-cTnT levels were detected in 20.2\% $(209 / 1,037)$ and $21.2 \%$ (170/801) patients, respectively. Detailed methodical descriptions and outcomes of interest are provided in Supplementary methods and results.

The rates of vascular events were higher in the high cTnl (Figure 1) and hs-cTnT (Figure 2) groups than in the respective normal groups. Multivariate Cox regression analyses revealed that patients in the high cTnl group had a significantly increased risk of MACCE compared to those in the normal cTnl group (hazard ratio [HR], 1.97; 95\% confidence interval [Cl], 1.13 to $3.44 ; P=0.016)$ (Figure 3 and Supplementary Table 5) after adjustment for confounders. Similarly, patients with high hs-cTnT levels had a significantly increased risk of MACCE compared to those with normal hs-cTnT levels (HR, 2.69; 95\% $\mathrm{Cl}, 1.44$ to $5.01 ; P=0.002$ ) (Figure 3 and Supplementary Table 5). In sensitivity analyses, prognostic values of both cTnl and hs-cTnT for predicting the risk of MACCE remained unchanged when cTn levels were analyzed using the overall cTn cutoff levels without sex-specific differences (Supplementary Table 6).

Regarding secondary outcomes, high cTnl and hs-cTnT levels were also significantly associated with the risk of vascular death (Figure 3 and Supplementary Table 5). High hs-cTnT levels were significantly associated with the risk of recurrent ischemic stroke ( $\mathrm{HR}, 2.62 ; 95 \% \mathrm{Cl}, 1.05$ to $6.57 ; P=0.039)$; this association was not observed for cTnl levels $(\mathrm{HR}, 1.40 ; 95 \% \mathrm{Cl}$, 
0.57 to $3.45 ; P=0.454$ ) (Figure 3 and Supplementary Table 5). No significant differences were observed in the risk of acute myocardial infarction between the normal and high troponin groups (Figure 3 and Supplementary Table 5).

This is the first real-world cohort study on troponin levels in ESUS and validates the finding of a recent clinical trial substudy that the troponin level is a predictor of the risk of vascular events in patients with ESUS. ${ }^{5}$ There is an urgent need to identify prognostic biomarkers related to potential cardiac dysfunction after ESUS. ${ }^{1}$ In a recent randomized trial on ESUS, a high hs-cTnT level was associated with increased cardiovascular events. ${ }^{5}$ Our data provide insights on the clinical significance of routine assessment of baseline troponin levels to predict vascular events after ESUS.

Our study has a few limitations. We were unable to perform all investigations for identifying PESs in ESUS, such as longterm cardiac monitoring, cardiac compound tomography, or magnetic resonance imaging. In addition, the predictive values of the two types of cTn were not compared. Since hs-cTnT assay has recently been introduced in clinical practice, patients who underwent hs-cTnT testing may have received more ad-
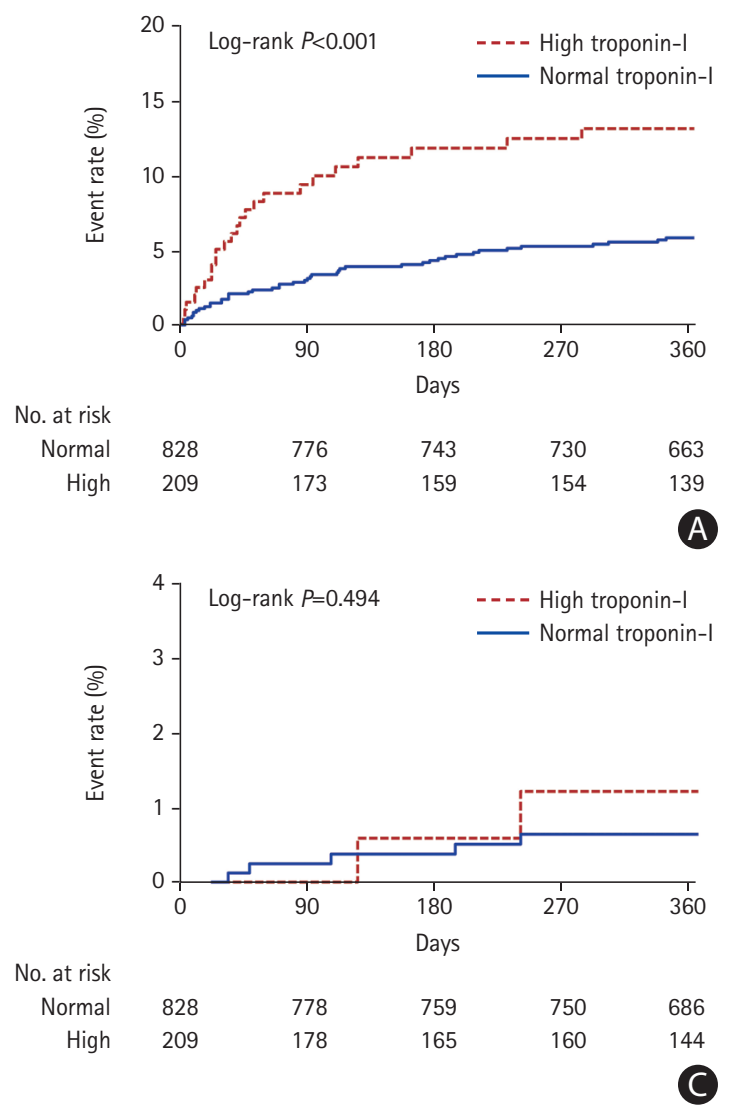

Figure 1. Vascular events based on conventional troponin I levels. (A) Major adverse cerebrovascular and cardiovascular event (MACCE), (B) recurrent stroke, (C) acute myocardial infarction, (D) vascular death. vanced treatment and better risk factor control than those who underwent cTnl testing. The risk of recurrent ischemic stroke was significantly associated with high hs-cTnT levels, but not with high cTnl levels. Further studies are needed to confirm whether hs-cTnT is superior to cTnl in predicting clinical outcomes and identify optimally tailored antithrombotics that reduce the risk of vascular events in patients with high cTn levels.

In conclusion, our findings suggest that high cTnl and hs-cTnT levels are significantly associated with MACCE and vascular death after ESUS. Our study also shows that high hs-cTnT levels are associated with a higher risk of recurrent ischemic stroke. Reducing the risk of MACCE after ESUS in individuals with high cTn levels is a challenge, and further studies are needed to improve patient care and clinical guidelines.

\section{Supplementary materials}

Supplementary materials related to this article can be found online at https://doi.org/10.5853/jos.2021.00010.
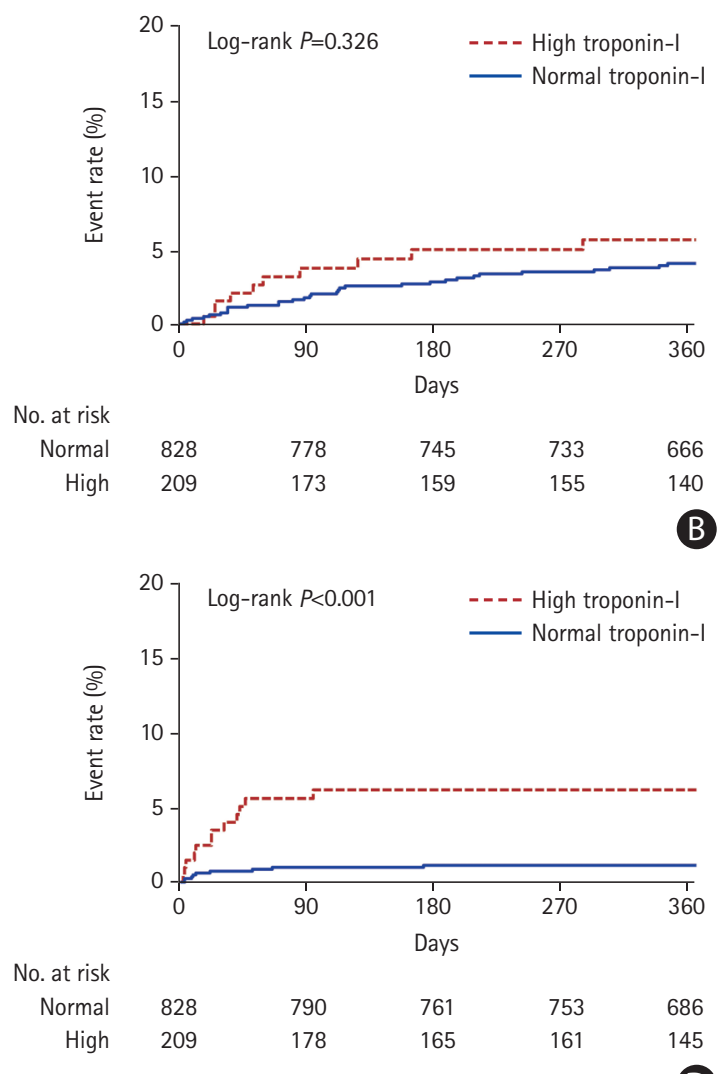

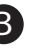

(D) 

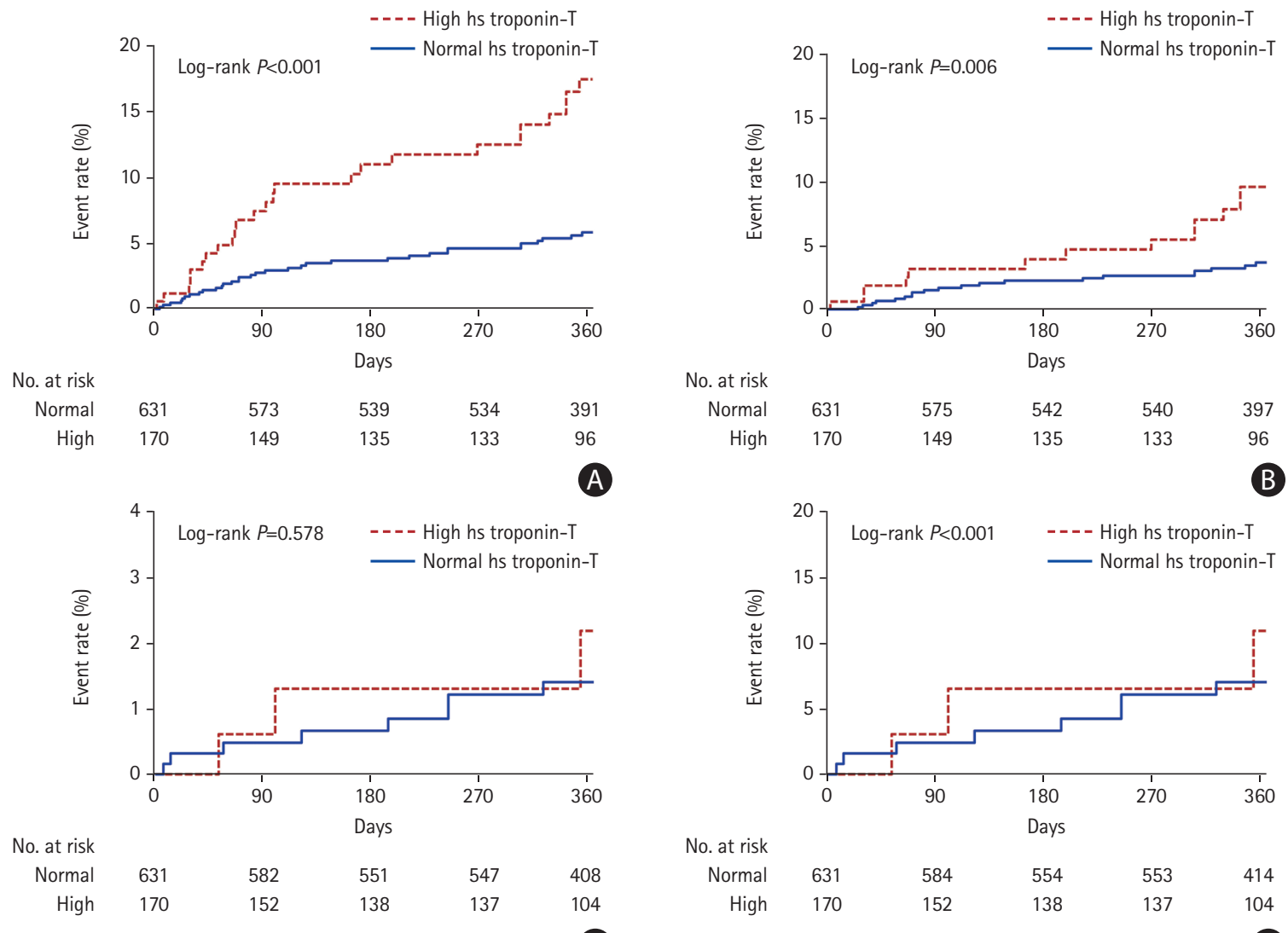

No. at risk

$\begin{array}{rccccc}\text { Normal } & 631 & 575 & 542 & 540 & 397 \\ \text { High } & 170 & 149 & 135 & 133 & 96\end{array}$

(A)

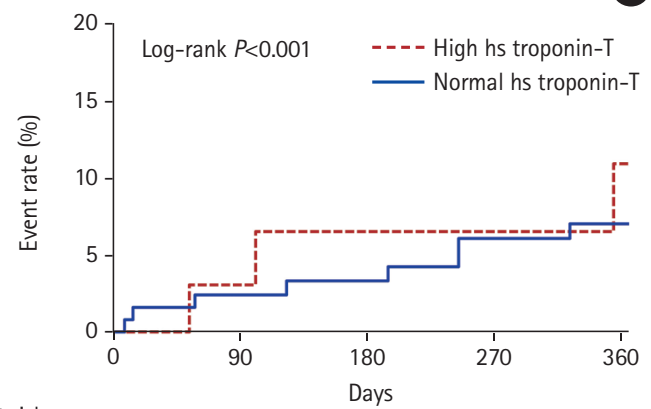

$\begin{array}{rrrrrr}\text { No. at risk } & & & & & \\ \text { Normal } & 631 & 584 & 554 & 553 & 414 \\ \text { High } & 170 & 152 & 138 & 137 & 104\end{array}$

C

(D)

Figure 2. Vascular events based on high-sensitivity troponin T levels. (A) Major adverse cerebrovascular and cardiovascular event (MACCE), (B) recurrent stroke, (C) acute myocardial infarction, (D) vascular death.

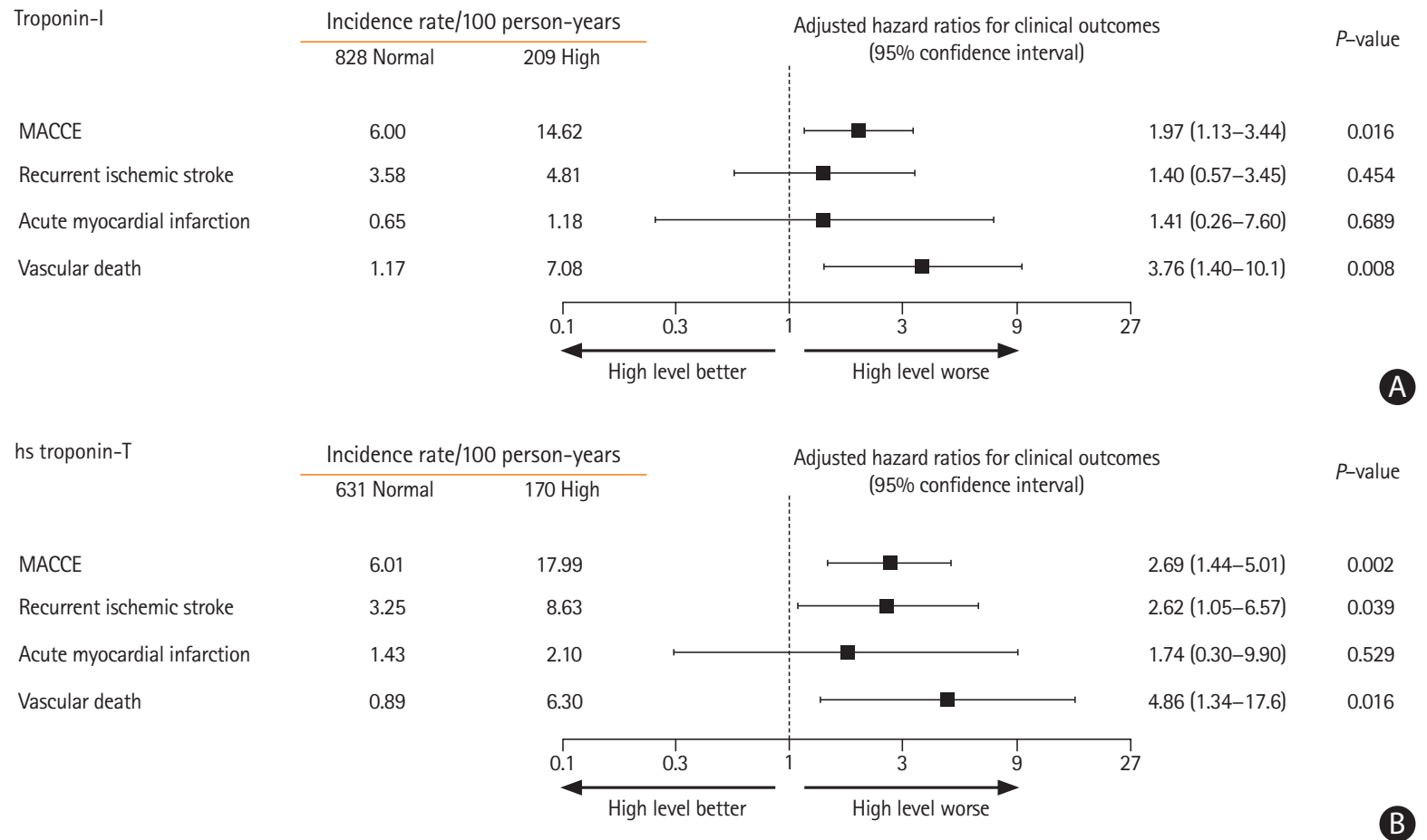

Figure 3. Association between cardiac troponin levels and clinical outcomes after embolic stroke of undetermined source. (A) Troponin I, (B) high-sensitivity troponin I (hs troponin-T). MACCE, major adverse cerebrovascular and cardiovascular event. 


\section{References}

1. Hart RG, Diener HC, Coutts $S B$, Easton JD, Granger $C B$, O'Donnell MJ, et al. Embolic strokes of undetermined source: the case for a new clinical construct. Lancet Neurol 2014;13: 429-438.

2. McCarthy CP, Raber I, Chapman AR, Sandoval Y, Apple FS, Mills NL, et al. Myocardial injury in the era of high-sensitivity cardiac troponin assays: a practical approach for clinicians. JAMA Cardiol 2019;4:1034-1042.

3. Merkler AE, Gialdini G, Murthy SB, Salehi Omran S, Moya A, Lerario MP, et al. Association between troponin levels and embolic stroke of undetermined source. J Am Heart Assoc 2017;6: e005905.

4. Ahn SH, Lee JS, Kim YH, Kim BJ, Kim YJ, Kang DW, et al. Prognostic significance of troponin elevation for long-term mortality after ischemic stroke. J Stroke 2017;19:312-322.

5. Scheitz JF, Pare G, Pearce LA, Mundl H, Peacock WF, Czlon- kowska $A$, et al. High-sensitivity cardiac troponin T for risk stratification in patients with embolic stroke of undetermined source. Stroke 2020;51:2386-2394.

Correspondence: Kang-Ho Choi

Department of Neurology, Chonnam National University Hospital, Chonnam National University Medical School, 42 Jebong-ro, Dong-gu, Gwangju 61469, Korea

Tel: +82-62-220-6137

Fax: +82-62-228-3461

E-mail: ckhchoikang@chonnam.ac.kr

https://orcid.org/0000-0001-8851-2104

Received: January 1, 2021

Revised: February 2, 2021

Accepted: February 3, 2021

This work was supported by a National Research Foundation of Korea grant funded by the Korean Government (NRF-2019M3A9E8020261, Kang-Ho Choi).

The authors have no financial conflicts of interest. 


\section{Supplementary methods}

\section{Subjects and study design}

In this single-center retrospective cohort study, we used a prospective registry of patients with acute ischemic stroke (AIS) who were admitted to our government-initiated comprehensive stroke center between January 2010 and May 2019. Patients were enrolled consecutively (1) if they had AIS and were admitted within 24 hours of onset of symptoms and (2) if the diagnosis of embolic stroke of undetermined source (ESUS) could be confirmed based on brain magnetic resonance imaging (MRI), 12-lead electrocardiography, cardiac monitoring for $\geq 24$ hours, transthoracic echocardiography, and computed tomography (CT) or magnetic resonance angiography for the evaluation of extracranial and intracranial arteries. ESUS was defined as non-lacunar embolic ischemic stroke without evidence of a major-risk cardioembolic source, presence of $\geq 50 \%$ stenosis of the lumen of an extracranial or intracranial artery supplying the area of brain ischemia, or stroke without other specific causes, such as arteritis, dissection, migraine/vasospasm, or drug misuse. Cardiac troponin (cTn) levels were measured immediately after admission. The exclusion criteria were as follows: (1) patients who did not undergo baseline serum troponin level assessment on admission or (2) patients lost to follow-up whose clinical outcomes could not be investigated (Supplementary Figure 1). Data on baseline characteristics, underlying risk factors of stroke, and laboratory findings were collected from all subjects.

Subjects were categorized into normal or high troponin groups according to their baseline cTn levels (Supplementary Figure 1). We used sex-specific 99th percentile upper reference limits (URL) of cTn I (cTnl) and high-sensitivity cTn T (hs-cTnT) as thresholds for classifying groups. cTnl levels were measured using a sensitive assay on an automated platform (Dimension Vista, Siemens, Berlin, Germany), with a reported 99th percentile value of $\leq 30 \mathrm{ng} / \mathrm{L}$ for healthy males and $\leq 15 \mathrm{ng} / \mathrm{L}$ for healthy females. hs-cTnT levels were measured using a highly sensitive assay on an automated platform (Elecsys, Roche Diagnostics, Basel, Switzerland), with a reported 99th percentile value of $\leq 20 \mathrm{ng} / \mathrm{L}$ for healthy males and $\leq 13 \mathrm{ng} / \mathrm{L}$ for healthy females (Supplementary Figure 1). The limits of detection of the cTnl and hs-cTnT assays were 10 and $5 \mathrm{ng} / \mathrm{L}$, respectively. At our center, hs-cTnT testing was performed since January 2015. Prior to January 2015, cTnl levels alone were tested. From January 2015, testing for a specific type of troponin (cTnl or hs-cTnT) depended on the physician's clinical judgment for each patient.

\section{Clinical assessment and outcome measurements}

The primary outcome measure was the first occurrence of ma- jor adverse cerebrovascular and cardiovascular events (MACCE; a composite of stroke, acute myocardial infarction [AMI], or death from a vascular cause) according to the baseline cTn levels over a 1-year period after ESUS. The key secondary outcomes included the incidence of recurrent ischemic stroke, intracerebral hemorrhage, $\mathrm{AMI}$, and death from a vascular cause. Recurrent stroke was defined as a sudden development of a new focal neurologic deficit or worsening of an existing focal neurologic deficit after the index stroke event, with evidence of attributable new stroke lesions (ischemic or hemorrhagic stroke) on brain imaging (CT or MRI). The severity of the neurological deficit was assessed using the National Institutes of Health Stroke Scale (NIHSS). Paradoxical embolism suggesting right-to-left shunt was defined as the presence of microembolic signals on the transcranial Doppler bubble test conducted during hospitalization for all patients. Information regarding clinical outcomes was obtained from all patients during hospitalization, during routine clinic visits, or via telephone interviews with patients or their caregivers. The information was assessed by trained stroke physicians or nurses.

\section{Statistical analysis}

Differences between the groups were analyzed using one-way analysis of variance, the Kruskal-Wallis test, or the Mann-Whitney $U$ test for continuous variables. The chi-square test or Fisher's exact test was used for non-continuous variables. Crude associations between cTn levels and the risk for clinical outcomes were analyzed over the study period using Kaplan-Meier curves with log-rank tests. Time-to-first event methods were used for the primary endpoint and for each secondary endpoint. Cox proportional hazard regression models were constructed to calculate the adjusted hazard ratios (HRs) and 95\% confidence intervals (Cls) for clinical outcomes, according to the baseline cTn levels. Adjustments were performed for age; sex; presence of hypertension, dyslipidemia, diabetes mellitus, coronary heart disease, and cancer; current smoking; prior history of stroke or transient ischemic attack; initial NIHSS score; initial blood pressure; leukocyte counts; hemoglobin, creatinine, and C-reactive protein levels; and administration of reperfusion therapy (intravenous alteplase or mechanical thrombectomy) based on their clinical significance. We conducted additional sensitivity analyses using the overall 99th percentile URL cutoff levels of $21 \mathrm{ng} / \mathrm{L}$ for cTnl and $15 \mathrm{ng} / \mathrm{L}$ for hs-cTnT without a sex-specific difference. Statistical significance was set at $P<0.05$ in all analyses. Statistical analyses were performed using IBM SPSS Statistics version 25.0 (IBM Corp., Armonk, NY, USA), SAS version 9.4 (SAS Institute Inc., Cary, NC, USA), and R software version 3.3.1 (R Foundation, Vienna, Austria). 


\section{Data availability and ethics statement}

All supporting study data can be obtained from the corresponding author on reasonable request from a qualified investigator. Our Institutional Review Board approved the present study. Written informed consent was obtained from all patients or their legal representatives for inclusion in the prospective stroke registry. We used the acronym "TOP-ESUS" to define our research, which stands for cTn levels and outcomes in patients with ESUS. All clinical and laboratory investigations described in this study were conducted in accordance with the principles outlined in the Declaration of Helsinki.

\section{Supplementary results}

\section{Patient characteristics}

In this study, we included 1,838 consecutive patients with ESUS (Supplementary Figure 1). A total of 1,037 (56.4\%) patients underwent cTnl testing and 801 (43.6\%) patients underwent hs-cTnT testing. The mean time from symptom onset to cTn testing was 11.9 hours. The mean follow-up duration was 10.6 months. The clinical and biochemical characteristics of patients according to cTn levels are presented in Supplementary Tables 1 and 2. Elevated cTnl levels were detected in $209(20.2 \%)$ of 1,037 patients tested for cTnl and elevated hs-cTnT levels were detected in $170(21.2 \%)$ of 801 patients tested for hs-cTnT. Patients who underwent cTnl testing were more likely to have hypertension, while those who underwent hs-cTnT testing were more likely to have dyslipidemia and old myocardial infarction (Supplementary Table 3). Detailed information on the incidence of ESUS and cTn assays performed each year at our center is provided in Supplementary Table 4.

\section{Clinical outcomes}

Among patients who underwent cTnl and hs-cTnT testing, 69 and 58 patients experienced MACCE during follow-up. The annual rates of MACCE were higher in the high cTnl and hs-cTnT groups than in the respective normal groups (14.62\% vs. $6.00 \%$ and $17.99 \%$ vs. $6.01 \%$, respectively) (Supplementary Table 5). Kaplan-Meier analyses showed that the risk of MACCE was significantly higher in the high troponin groups than in the respective normal groups, regardless of the type of troponin test performed (log-rank $P<0.001$ ) (Figures 1 and 2). In multivariate Cox regression analyses, patients in the high cTnl group had a significantly increased risk of MACCE com- pared to those in the normal cTnl group $(\mathrm{HR}, 1.97 ; 95 \% \mathrm{Cl}$, 1.13 to $3.44 ; P=0.016$ ) (Figure 3 and Supplementary Table 5) after adjustment for confounders. Similarly, patients with high hs-cTnT levels had a significantly increased risk of MACCE compared to those with normal hs-cTnT levels (HR, 2.69; 95\% $\mathrm{Cl}, 1.44$ to $5.01 ; P=0.002$ ) (Figure 3 and Supplementary Table 5).

Regarding secondary outcomes, high cTnl and hs-cTnT levels were significantly associated with the risk of vascular death in patients with ESUS (HR, 3.76; $95 \% \mathrm{Cl}, 1.40$ to $10.1 ; P=0.008$ and $\mathrm{HR}, 4.86 ; 95 \% \mathrm{Cl}_{1} 1.34$ to $17.6 ; P=0.016$, respectively) (Figure 3 and Supplementary Table 5). High hs-cTnT levels were significantly associated with the risk of recurrent ischemic stroke $(\mathrm{HR}, 2.62 ; 95 \% \mathrm{Cl}, 1.05$ to $6.57 ; P=0.039)$; this association was not observed for cTnl levels $(\mathrm{HR}, 1.40 ; 95 \% \mathrm{Cl}$, 0.57 to 3.45 ; $P=0.454$ ) (Figure 3 and Supplementary Table 5). No significant differences were observed in the risk of hemorrhagic stroke and AMI between the normal and high troponin groups (Figure 2 and Supplementary Table 5).

In the sensitivity analysis, we evaluated the association between elevated cTn levels and clinical outcomes using the overall cTn cutoff levels without sex-specific differences. The prognostic values of both cTnl and hs-cTnT for predicting the risk of MACCE were similar (Supplementary Table 6). High cTnl and hs-cTnT levels were significantly associated with a higher risk of MACCE $(H R, 2.40 ; 95 \% \mathrm{Cl}, 1.39$ to $4.14 ; P=0.002$ and $\mathrm{HR}, 3.58 ; 95 \% \mathrm{Cl}, 1.87$ to $6.86 ; P<0.001$, respectively) and vascular death in patients with ESUS (Supplementary Table 6). The high hs-cTnT group, identified using the overall hs-cTnT cutoff levels, had a higher risk of recurrent ischemic stroke than the normal hs-cTnT group (HR, 2.96; $95 \% \mathrm{Cl}, 1.22$ to 7.16; $P=0.016$ ) (Supplementary Table 6). 
Supplementary Table 1. Baseline characteristics according to baseline cardiac troponin levels

\begin{tabular}{|c|c|c|c|c|c|c|}
\hline \multirow{2}{*}{ Characteristic } & \multicolumn{3}{|c|}{ Conventional troponin I } & \multicolumn{3}{|c|}{ High-sensitivity troponin T } \\
\hline & Normal $(n=828)$ & High $(n=209)$ & $P$ & Normal $(n=631)$ & High $(n=170)$ & $P$ \\
\hline Age in years & $63.8 \pm 13.4$ & $71.0 \pm 11.7$ & $<0.001$ & $63.6 \pm 13.5$ & $73.4 \pm 10.8$ & $<0.001$ \\
\hline Male sex & $535(64.6)$ & $95(45.5)$ & $<0.001$ & $432(68.5)$ & $73(42.9)$ & $<0.001$ \\
\hline \multicolumn{7}{|l|}{ Comorbidities } \\
\hline Hypertension & $434(52.4)$ & $124(59.3)$ & 0.087 & $288(45.6)$ & $98(57.6)$ & 0.007 \\
\hline Diabetes mellitus & $201(24.3)$ & $55(26.3)$ & 0.602 & $147(23.3)$ & $49(28.8)$ & 0.165 \\
\hline Dyslipidemia & $120(14.5)$ & $29(13.9)$ & 0.907 & $121(19.2)$ & $23(13.5)$ & 0.112 \\
\hline Cancer & $100(12.1)$ & $34(16.3)$ & 0.134 & $56(8.9)$ & $25(14.7)$ & 0.036 \\
\hline Current smoking & $211(25.5)$ & $28(13.4)$ & $<0.001$ & $147(23.3)$ & $17(10.0)$ & $<0.001$ \\
\hline Coronary heart disease & $46(5.6)$ & $17(8.1)$ & 0.218 & $43(6.8)$ & $22(12.9)$ & 0.015 \\
\hline Prior stroke or TIA & $149(18.0)$ & 27 (12.9) & 0.100 & $101(16.0)$ & $36(21.2)$ & 0.140 \\
\hline \multicolumn{7}{|l|}{ Cardiac markers } \\
\hline Troponin (ng/L) & $14.4 \pm 3.5$ & $285.2 \pm 794.3$ & $<0.001$ & $8.9 \pm 5.7$ & $42.4 \pm 53.0$ & $<0.001$ \\
\hline NT-pro BNP (ng/mL) & $0.9 \pm 3.3$ & $4.5 \pm 9.2$ & $<0.001$ & $0.9 \pm 3.4$ & $2.6 \pm 6.5$ & 0.054 \\
\hline CK-MB (ng/mL) & $3.9 \pm 9.0$ & $7.0 \pm 16.6$ & 0.335 & $2.0 \pm 3.7$ & $3.1 \pm 2.4$ & 0.004 \\
\hline Myoglobin $(\mu \mathrm{g} / \mathrm{mL})$ & $0.2 \pm 0.3$ & $0.5 \pm 1.3$ & 0.158 & $0.2 \pm 0.4$ & $0.3 \pm 0.6$ & 0.555 \\
\hline \multicolumn{7}{|l|}{ Biochemical variables } \\
\hline Total cholesterol (mg/dL) & $180.4 \pm 43.1$ & $173.1 \pm 48.9$ & 0.057 & $177.3 \pm 43.3$ & $173.4 \pm 45.7$ & 0.333 \\
\hline LDL-C (mg/dL) & $118.2 \pm 54.3$ & $111.0 \pm 41.9$ & 0.054 & $113.4 \pm 57.8$ & $107.8 \pm 38.0$ & 0.164 \\
\hline Triglyceride (mg/dL) & $116.7 \pm 70.3$ & $108.9 \pm 59.5$ & 0.108 & $118.6 \pm 70.0$ & $115.7 \pm 60.1$ & 0.614 \\
\hline $\mathrm{HDL}-\mathrm{C}(\mathrm{mg} / \mathrm{dL})$ & $45.1 \pm 12.1$ & $43.9 \pm 14.3$ & 0.330 & $46.0 \pm 16.9$ & $45.4 \pm 14.3$ & 0.664 \\
\hline Fasting blood sugar (mg/dL) & $122.1 \pm 46.3$ & $127.8 \pm 51.5$ & 0.154 & $124.8 \pm 48.1$ & $119.5 \pm 43.5$ & 0.230 \\
\hline Glycated hemoglobin (\%) & $6.1 \pm 1.3$ & $6.1 \pm 1.2$ & 0.861 & $6.2 \pm 1.4$ & $6.2 \pm 1.3$ & 0.684 \\
\hline Hemoglobin (g/dL) & $13.7 \pm 1.9$ & $12.7 \pm 2.0$ & $<0.001$ & $13.9 \pm 1.9$ & $12.9 \pm 1.9$ & $<0.001$ \\
\hline Leukocyte counts $\left(\times 10^{9} / \mathrm{L}\right)$ & $8.7 \pm 3.1$ & $9.0 \pm 3.4$ & 0.309 & $8.4 \pm 3.0$ & $8.1 \pm 2.7$ & 0.369 \\
\hline Creatinine (mg/dL) & $0.8 \pm 0.6$ & $1.0 \pm 1.1$ & 0.016 & $0.9 \pm 0.4$ & $1.1 \pm 1.1$ & 0.009 \\
\hline C-reactive protein (mg/dL) & $0.7 \pm 2.0$ & $1.2 \pm 2.5$ & 0.003 & $0.7 \pm 2.2$ & $1.2 \pm 2.0$ & 0.005 \\
\hline Initial NIHSS & $2(0-7)$ & $3(1-9)$ & 0.008 & $2(0-5)$ & $3(1-8)$ & 0.003 \\
\hline Systolic blood pressure (mm Hg) & $137.8 \pm 23.0$ & $136.9 \pm 23.7$ & 0.606 & $136.4 \pm 22.6$ & $139.3 \pm 21.6$ & 0.160 \\
\hline Diastolic blood pressure $(\mathrm{mm} \mathrm{Hg})$ & $83.6 \pm 13.3$ & $81.4 \pm 14.5$ & 0.039 & $82.5 \pm 14.1$ & $81.8 \pm 15.4$ & 0.601 \\
\hline Intravenous alteplase & $145(17.5)$ & $25(12.0)$ & 0.067 & $64(10.1)$ & $21(12.4)$ & 0.490 \\
\hline Mechanical thrombectomy & $56(6.8)$ & $16(7.7)$ & 0.763 & $61(9.7)$ & $17(10.0)$ & 1.000 \\
\hline Lesion territory & & & 0.954 & & & 1.000 \\
\hline Anterior circulation & $547(66.1)$ & $137(65.6)$ & & $416(65.9)$ & $112(65.9)$ & \\
\hline Posterior circulation & $281(33.9)$ & $72(34.4)$ & & $215(34.1)$ & $58(34.1)$ & \\
\hline
\end{tabular}

Values are presented as mean \pm standard deviation, number (\%), or median (interquartile range).

TIA, transient ischemic attack; NT-pro BNP, N-terminal pro B-type natriuretic peptide; CK-MB, creatine kinase myocardial band; LDL-C, low-density lipoprotein cholesterol; HDL-C, high-density lipoprotein cholesterol; NIHSS, National Institutes of Health Stroke Scale. 
Supplementary Table 2. Transcranial and echocardiographic findings according to cardiac troponin levels

\begin{tabular}{|c|c|c|c|c|c|c|}
\hline \multirow{2}{*}{ Variable } & \multicolumn{3}{|c|}{ Conventional troponin I } & \multicolumn{3}{|c|}{ High-sensitivity troponin T } \\
\hline & Normal $(n=828)$ & High $(n=209)$ & $P$ & Normal $(n=631)$ & High $(n=170)$ & $P$ \\
\hline \multicolumn{7}{|l|}{ Transcranial Doppler } \\
\hline Paradoxical embolism & $301(36.4)$ & $72(34.4)$ & 0.781 & $256(40.6)$ & $68(40.0)$ & 0.963 \\
\hline \multicolumn{7}{|l|}{ Holter monitoring } \\
\hline Atrial tachycardia & $70(8.5)$ & $29(13.9)$ & 0.024 & $44(7.0)$ & $18(10.6)$ & 0.160 \\
\hline \multicolumn{7}{|l|}{ Transthoracic echocardiography } \\
\hline Old myocardial infarction & $29(3.5)$ & $19(9.1)$ & 0.001 & $36(5.7)$ & $21(12.4)$ & 0.005 \\
\hline Left atrial enlargement & $8(1.0)$ & $9(4.3)$ & 0.002 & $6(1.0)$ & $5(2.9)$ & 0.108 \\
\hline Left ventricular hypertrophy & $23(2.8)$ & $18(8.6)$ & $<0.001$ & $19(3.0)$ & $13(7.6)$ & 0.012 \\
\hline Ejection fraction $<50 \%$ & $88(10.6)$ & $28(13.4)$ & 0.311 & $79(12.5)$ & 32 (18.8) & 0.047 \\
\hline Aortic valve stenosis & $62(7.5)$ & $33(15.8)$ & 0.002 & $67(10.6)$ & $20(11.8)$ & 0.285 \\
\hline Pulmonary hypertension & $160(19.3)$ & $68(32.5)$ & $<0.001$ & $112(17.7)$ & $59(34.7)$ & $<0.001$ \\
\hline Diastolic dysfunction & $613(74.0)$ & $159(76.1)$ & 0.076 & $482(76.4)$ & 137 (80.6) & 0.016 \\
\hline
\end{tabular}

Values are presented as number (\%). 
Supplementary Table 3. Baseline characteristics according to troponin type

\begin{tabular}{|c|c|c|c|c|}
\hline Characteristic & Troponin-I $(n=1,037)$ & High-sensitivity troponin- $\mathrm{T}(\mathrm{n}=801)$ & Total $(n=1,838)$ & $P$ \\
\hline Age in years & $65.2 \pm 13.4$ & $65.7 \pm 13.6$ & $65.4 \pm 13.5$ & 0.433 \\
\hline Male sex & $630(60.8)$ & $505(63.0)$ & $1,135(61.8)$ & 0.340 \\
\hline \multicolumn{5}{|l|}{ Risk factors } \\
\hline Hypertension & $558(53.8)$ & $386(48.2)$ & $944(51.4)$ & 0.019 \\
\hline Diabetes mellitus & $256(24.7)$ & $196(24.5)$ & $452(24.6)$ & 0.958 \\
\hline Dyslipidemia & $149(14.4)$ & $144(18.0)$ & $293(15.9)$ & 0.042 \\
\hline Cancer & $134(12.9)$ & $81(10.1)$ & $215(11.7)$ & 0.074 \\
\hline Current smoking & $239(23.0)$ & $164(20.5)$ & $403(21.9)$ & 0.206 \\
\hline Coronary heart disease & $63(6.1)$ & $65(8.1)$ & $128(7.0)$ & 0.107 \\
\hline Prior stroke or TIA & $176(17.0)$ & $137(17.1)$ & $313(17.0)$ & 0.991 \\
\hline \multicolumn{5}{|l|}{ Cardiac markers } \\
\hline NT-pro BNP (ng/mL) & $1.5 \pm 5.1$ & $1.4 \pm 4.6$ & $1.5 \pm 4.9$ & 0.707 \\
\hline CK-MB (ng/mL) & $5.1 \pm 12.6$ & $2.3 \pm 3.5$ & $2.8 \pm 6.5$ & 0.051 \\
\hline Myoglobin $(\mu \mathrm{g} / \mathrm{mL})$ & $0.3 \pm 0.8$ & $0.2 \pm 0.5$ & $0.3 \pm 0.7$ & 0.625 \\
\hline \multicolumn{5}{|l|}{ Biochemical variables } \\
\hline Total cholesterol (mg/dL) & $178.9 \pm 44.4$ & $176.5 \pm 43.8$ & $177.9 \pm 44.1$ & 0.267 \\
\hline LDL-C (mg/dL) & $116.8 \pm 52.2$ & $112.2 \pm 54.1$ & $114.8 \pm 53.1$ & 0.090 \\
\hline Triglyceride (mg/dL) & $115.2 \pm 68.3$ & $118.0 \pm 68.0$ & $116.3 \pm 68.2$ & 0.403 \\
\hline $\mathrm{HDL}-\mathrm{C}(\mathrm{mg} / \mathrm{dL})$ & $44.8 \pm 12.6$ & $45.8 \pm 16.4$ & $45.3 \pm 14.3$ & 0.190 \\
\hline Fasting blood sugar (mg/dL) & $123.2 \pm 47.4$ & $123.7 \pm 47.1$ & $123.4 \pm 47.3$ & 0.839 \\
\hline Glycated hemoglobin (\%) & $6.1 \pm 1.3$ & $6.2 \pm 1.4$ & $6.1 \pm 1.3$ & 0.097 \\
\hline Initial NIHSS & $2(1-7)$ & $2(0-6)$ & $2(1-7)$ & 0.064 \\
\hline Reperfusion therapies & $203(19.6)$ & $131(16.4)$ & $334(18.2)$ & 0.086 \\
\hline Paradoxical embolism & $373(36.0)$ & $324(40.4)$ & $697(37.9)$ & 0.094 \\
\hline Atrial tachycardia & $99(9.5)$ & $62(7.7)$ & $161(8.8)$ & 0.202 \\
\hline \multicolumn{5}{|l|}{ Transthoracic echocardiography } \\
\hline Old myocardial infarction & $48(4.6)$ & $57(7.1)$ & $105(5.7)$ & 0.029 \\
\hline Left atrial enlargement & $17(1.6)$ & $11(1.4)$ & $28(1.5)$ & 0.787 \\
\hline Left ventricular hypertrophy & $41(4.0)$ & $32(4.0)$ & $73(4.0)$ & 1.000 \\
\hline Aortic valve stenosis & $95(9.2)$ & 87 (10.9) & $182(9.9)$ & 0.631 \\
\hline Pulmonary hypertension & $228(22.0)$ & $171(21.3)$ & $399(21.7)$ & 0.970 \\
\hline Diastolic dysfunction & $772(74.4)$ & 619 (77.3) & $1,391(75.7)$ & 0.149 \\
\hline
\end{tabular}

Values are presented as mean \pm standard deviation, number $(\%)$, or median (interquartile range).

TIA, transient ischemic attack; NT-pro BNP, N-terminal pro B-type natriuretic peptide; CK-MB, creatine kinase myocardial band; LDL-C, low-density lipoprotein cholesterol; HDL-C, high-density lipoprotein cholesterol; NIHSS, National Institutes of Health Stroke Scale. 
Supplementary Table 4. The number of enrolled patients during cTn assays performed each year in this study

\begin{tabular}{|c|c|c|c|c|c|}
\hline Year & All AIS & ESUS & Enrolled & cTnl & hs-cTnT \\
\hline 2010 & 1,137 & 199 & 189 & 189 & - \\
\hline 2011 & 1,071 & 141 & 134 & 134 & - \\
\hline 2012 & 1,146 & 150 & 145 & 145 & - \\
\hline 2013 & 1,118 & 201 & 196 & 196 & - \\
\hline 2014 & 1,083 & 218 & 211 & 211 & - \\
\hline 2015 & 1,070 & 224 & 220 & 73 & 147 \\
\hline 2016 & 1,089 & 215 & 209 & 25 & 184 \\
\hline 2017 & 974 & 212 & 207 & 29 & 178 \\
\hline 2018 & 985 & 211 & 198 & 21 & 177 \\
\hline $2019^{*}$ & 456 & 136 & 129 & 14 & 115 \\
\hline Total & 10,255 & 1,907 & 1,838 & 1,037 & 801 \\
\hline
\end{tabular}

cTn, cardiac troponin; AIS, acute ischemic stroke; ESUS, embolic stroke of undetermined source (admitted within 24 hours of the onset of symptoms); hs-cTnT, high-sensitivity cTn T.

*Until May 2019.

Supplementary Table 5. Event rates and association estimates from Cox proportional hazard model according to baseline cardiac troponin levels

\begin{tabular}{|c|c|c|c|c|c|c|}
\hline \multirow{2}{*}{ Clinical outcome } & \multicolumn{3}{|c|}{ Conventional troponin I } & \multicolumn{3}{|c|}{ High-sensitivity troponin T } \\
\hline & Normal $(n=828)$ & High $(n=209)$ & $P$ & Normal $(n=631)$ & High $(n=170)$ & $P$ \\
\hline \multicolumn{7}{|l|}{ Primary outcome } \\
\hline \multicolumn{7}{|l|}{ MACCE } \\
\hline Incidence per 100 person-years & 6.00 & 14.62 & & 6.01 & 17.99 & \\
\hline Adjusted HR $(95 \% \mathrm{Cl})^{*}$ & Reference & $1.97(1.13-3.44)$ & 0.016 & Reference & $2.69(1.44-5.01)$ & 0.002 \\
\hline \multicolumn{7}{|l|}{ Secondary outcomes } \\
\hline \multicolumn{7}{|l|}{ Recurrent ischemic stroke } \\
\hline Incidence per 100 person-years & 3.58 & 4.81 & & 3.25 & 8.63 & \\
\hline Adjusted HRs $(95 \% \mathrm{Cl})^{*}$ & Reference & $1.40(0.57-3.45)$ & 0.454 & Reference & $2.62(1.05-6.57)$ & 0.039 \\
\hline \multicolumn{7}{|l|}{ Intracerebral hemorrhage } \\
\hline Incidence per 100 person-years & 0.52 & 1.19 & & 0.36 & 0.70 & \\
\hline Adjusted HRs $(95 \% \mathrm{Cl})^{*}$ & Reference & $4.12(0.60-28.2)$ & 0.149 & Reference & $2.03(0.14-29.0)$ & 0.600 \\
\hline \multicolumn{7}{|l|}{ Acute myocardial infarction } \\
\hline Incidence per 100 person-years & 0.65 & 1.18 & & 1.43 & 2.10 & \\
\hline Adjusted HRs $(95 \% \mathrm{CI})^{*}$ & Reference & $1.41(0.26-7.60)$ & 0.689 & Reference & $1.74(0.30-9.90)$ & 0.529 \\
\hline \multicolumn{7}{|l|}{ Death from vascular causes } \\
\hline Incidence per 100 person-years & 1.17 & 7.08 & & 0.89 & 6.30 & \\
\hline Adjusted HR $(95 \% \mathrm{Cl})^{*}$ & Reference & $3.76(1.40-10.1)$ & 0.008 & Reference & $4.86(1.34-17.6)$ & 0.016 \\
\hline
\end{tabular}

MACCE, major adverse cerebrovascular and cardiovascular events; $\mathrm{HR}$, hazard ratio; $\mathrm{Cl}$, confidence interval.

*Adjusted variables: age, sex, hypertension, dyslipidemia, diabetes mellitus, coronary heart disease, cancer, current smoking, prior history of stroke or transient ischemic attack, initial National Institutes of Health Stroke Scale score, initial blood pressure, hemoglobin, leukocyte counts, creatinine, C-reactive protein, and reperfusion therapies. 
Supplementary Table 6. Association between high cardiac troponin levels using the overall 99th percentile upper reference cutoff level and clinical outcomes

\begin{tabular}{|c|c|c|c|c|c|}
\hline \multirow{2}{*}{ Independent variable } & \multicolumn{5}{|c|}{ Adjusted HR $(95 \% \mathrm{Cl})^{*}$} \\
\hline & MACCE & Recurrent IS & $\mathrm{ICH}$ & $\mathrm{AMI}$ & Vascular death \\
\hline \multicolumn{6}{|c|}{ Conventional troponin-I } \\
\hline Normal & Reference & Reference & Reference & Reference & Reference \\
\hline High & $2.40(1.39-4.14)$ & $1.80(0.78-4.10)$ & $2.86(0.45-18.19)$ & $3.11(0.27-35.12)$ & $3.52(1.23-10.02)$ \\
\hline \multicolumn{6}{|c|}{ High-sensitivity troponin-T } \\
\hline Normal & Reference & Reference & Reference & Reference & Reference \\
\hline High & $3.58(1.87-6.86)$ & $2.96(1.22-7.16)$ & $1.96(0.14-27.48)$ & $1.86(0.42-8.08)$ & $4.92(1.34-17.98)$ \\
\hline
\end{tabular}

$\mathrm{HR}$, hazard ratio; $\mathrm{Cl}$, confidence interval; MACCE, major adverse cerebrovascular and cardiovascular events; IS, ischemic stroke; ICH, intracerebral hemorrhage; $A M I$, acute myocardial infarction.

*Adjusted variables: age, sex, hypertension, dyslipidemia, diabetes mellitus, coronary heart disease, cancer, current smoking, prior history of stroke or transient ischemic attack, initial National Institutes of Health Stroke Scale score, initial blood pressure, hemoglobin, leukocyte counts, creatinine, C-reactive protein, and reperfusion therapies.

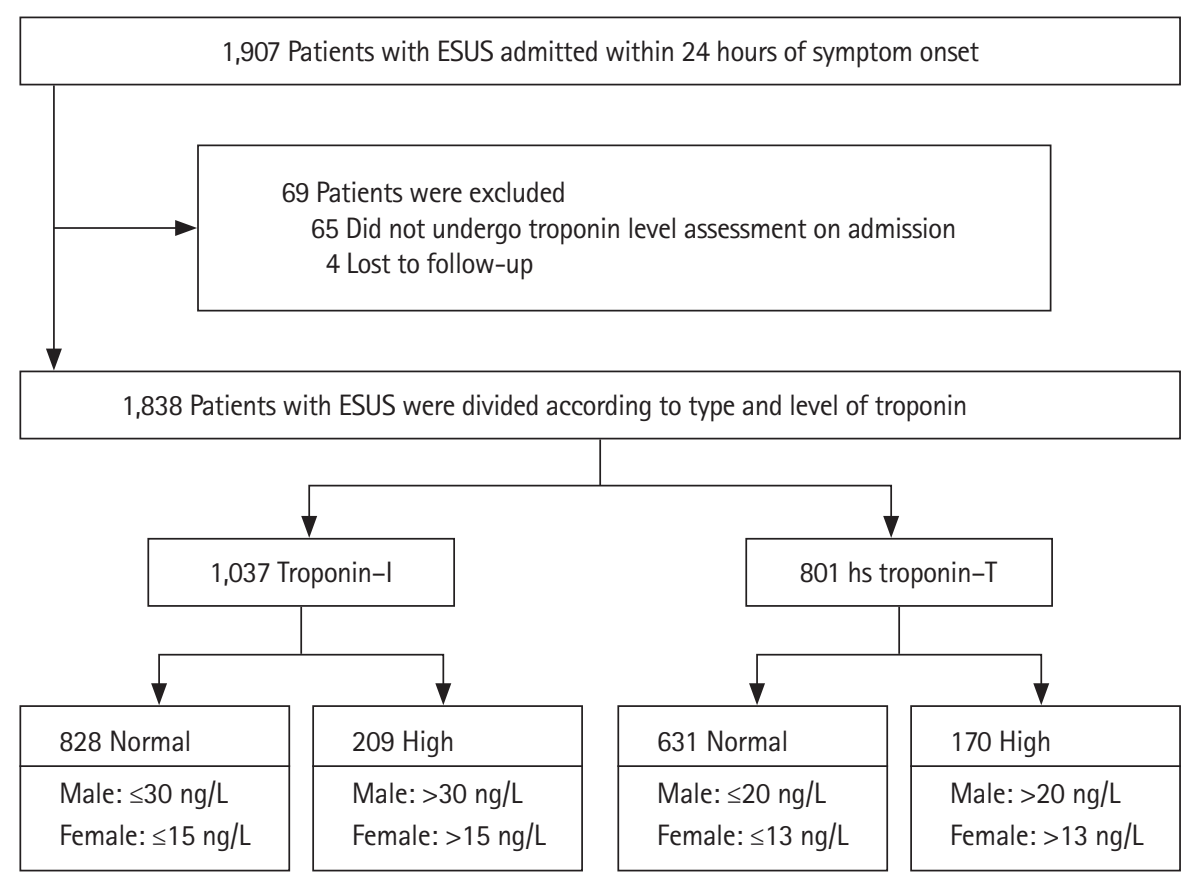

Supplementary Figure 1. Subject enrollment and clinical outcome measures. ESUS, embolic stroke of undetermined source; hs, high-sensitivity. 